08

\title{
Спонтанные переходы в состояние высокой проводимости в композитных пленках поливинилхлорида
}

\author{
(C) Т.В. Власова, С.И. Расмагин
}

Институт общей физики им. А.М. Прохорова РАН, 119991 Москва, Россия e-mail: rasmas123@yandex.ru

Поступило в Редакцию 4 апреля 2018 г.

В окончательной редакции 26 октября 2018 г.

Принято к публикации 9 июня 2019 г.

Проанализированы результаты экспериментальных исследований аномальной проводимости в пленках специально синтезированного сополимера. В макромолекулы поливинилхлорида были внедрены полиацетиленовые молекулярные фрагменты с переменной концентрацией. Было экспериментально установлено, что в таких образцах происходят спонтанные и стимулированные скачки проводимости на 13 порядков, а жизнь каждого из этих состояний может быть очень большой от нескольких минут до суток. Предложена качественная модель, описывающая аномальное поведение проводимости композита поливинилхлорида, включающее механизмы стабилизации состояния высокой проводимости, условия развития неустойчивости в переходах между состояниями, а также причины долгоживущего состояния высокой проводимости даже при отключенном приложенном напряжении. Рассмотрены также простые численные оценки, подтверждающие реальность предлагаемых механизмов.

Ключевые слова: поливинилхлорид, полимерные пленки, дегидрохлорирование, аномальная проводимость.

DOI: $10.21883 /$ JTF.2019.12.48494.220-18

\section{Введение}

В последнее время активно проводятся исследования специфики электрических свойств проводящих (содержащих двойные сопряженные связи) [1,2], широкозонных полимеров [3] и композитных материалов на их основе $[4,5]$. Весь этот класс совместно образуют совокупность элементов с весьма широким диапазоном проводимости от почти идеального металла до идеального изолятора. В отличие от упорядоченных проводников полимерные материалы и композиты на их основе демонстрируют высокую специфичность проводящих свойств. Например, получены радикальные изменения электропроводности при внешних воздействиях, которые открывают широкие перспективы создания новых приборов полимерной электроники типа ячеек памяти, различных датчиков и схемотехнических полимерных элементов. Некоторые элементы такой электроники, такие как органические светодиоды, уже нашли свое место на рынке, а разработки в области литий-полимерных батарей уже превосходят по ряду параметров известные ранее типы. Тем не менее на сегодняшний день многие явления и характеристики полимерных композитов мало исследованы, поскольку они имеют сложную неупорядоченную фрактальную супрамолекулярную структуpy $[6,7]$, в которой физические процессы, такие как электропроводность и химическое строение тесно переплетены. В частности, к таким сложным явлениям можно отнести переключения проводящих состояний в относительно толстых пленочных образцах пластикатов и композитов на основе поливинилхлорида, исследо- ванные в ряде наших публикаций [8-10]. О важности и абсолютной величине эффекта переключений можно судить на примере сополимера винилхлорида и полиацетилена $[11,12]$, где были обнаружены спонтанные скачки проводимости на 13 порядков величины. Подобные явления можно рассматривать как аналоги андерсоновского перехода металл-диэлектрик в неупорядоченных средах или пытаться использовать другие многочисленные теории переключения проводимости неупорядоченных материалов, в частности, полимеров, включая теории возникновения пробоя. Среди наиболее популярных моделей проводимости можно отметить многочисленные исследования полимерной проводимости за счет формирования комплексов переноса заряда [13] - полимерных донорно-акцепторных комплексов, обеспечивающих проводимость полупроводникового уровня пленочных образцов. Другой подробно исследованный и описанный в монографии [14] и обзоре [15] физический механизм проводимости в неупорядоченных средах - прыжковая проводимость, разработанная для слабо легированных полупроводников. Описанная проводимость возникает за счет „прыжков“ носителей заряда между атомами примеси (примесная проводимость и моттовский переход металл-диэлектрик), которая отчасти применима для описания некоторых случаев переключения полимерной проводимости. При этом „прыжки“ могут быть связаны с термической активацией („некогерентный“ надбарьерный механизм [16]) и с подбарьерным туннелированием („когерентныйс квантовый механизм [17]). Известны многочисленные теории механизмов проводимости в тонких нанометровых пленках, где основную 
роль играют контактные эффекты, связанные с инжекцией носителей и возникновением токов, ограниченных пространственным зарядом [18,19]. Также представлены различные механизмы переключений проводимости, наблюдаемые в тонких пленках широкозонных полимеров [3]. Обычно аномально высокая проводимость пленок аморфных полупроводников связана с возникновением тонких проводящих каналов, расположенных перпендикулярно поверхности [20-23]. В некоторых исследованиях специально отмечалось существование граничной критической толщины $L_{\mathrm{cr}}$, при превышении которой скачки проводимости не наблюдались. Практически наблюдаемая толщина $L_{\mathrm{cr}}$ ограничивается субмикронным или даже нанометровым диапазоном [3]. В отличие от перечисленных данных в наших работах исследовались относительно толстые пленки с толщиной от десятков до сотен микрон. На наш взгляд, существующие теоретические модели по большей части построены ad hoc и не описывают наблюдаемые нами аномальные явления спонтанных переключений проводимости, устойчивость и значительную продолжительность времени жизни состояния высокой проводимости (СВП), относительно быстрое переключение состояний проводимости в полимерных композитах на базе поливинилхлорида (ПВХ).

\section{Экспериментальная часть}

В результате исследований проводимости полимерных композитов $[11,12]$ нами было обнаружено, что в образцах композита, приготовленных с использованием частично дегидрохлорированного ПВХ, проявляется аномальный эффект. Суть эффекта заключается в гигантских скачкообразных переключениях состояний проводимости, которые составляли до 12 порядков величины: от уровня сопротивления изолятора порядка $10^{12} \Omega$ до единиц и долей $\Omega$. С другой стороны, химическая структура макромолекул дегидрохлорированного ПВХ хорошо известна. Сам процесс дегидрохлорирования (термолиза) фактически эквивалентен одному из известных механизмов термического старения изоляционных материалов. Во время термолиза раствора ПВХ при температуре $t=195^{\circ} \mathrm{C}$ происходит отщепление молекулы $\mathrm{HCl}$ от макромолекулы ПВХ и в результате образуется звено цепи макромолекулы, содержащее сопряженную двойную связь между атомами углерода $-\mathrm{C}=\mathrm{C}-$. В продолжении процесса термолиза выделяются молекулы $\mathrm{HCl}$,

\section{Conductor \\ Insulator

\begin{tabular}{|c|c|}
\hline & $\mathrm{Cl}$ \\
\hline$-\mathrm{CH}=\mathrm{CH}-\mathrm{CH}=\mathrm{CH}-\mathrm{CH}=\mathrm{CH}$ & $-\mathrm{CH}_{2}-\mathrm{CH}-\mathrm{CH}_{2}-\mathrm{CH}-\mathrm{CH}_{2}$ \\
\hline
\end{tabular}

Рис. 1. Структура макромолекул специально синтезированного полимера (в реальных образцах макромолекулы ориентированы хаотически).

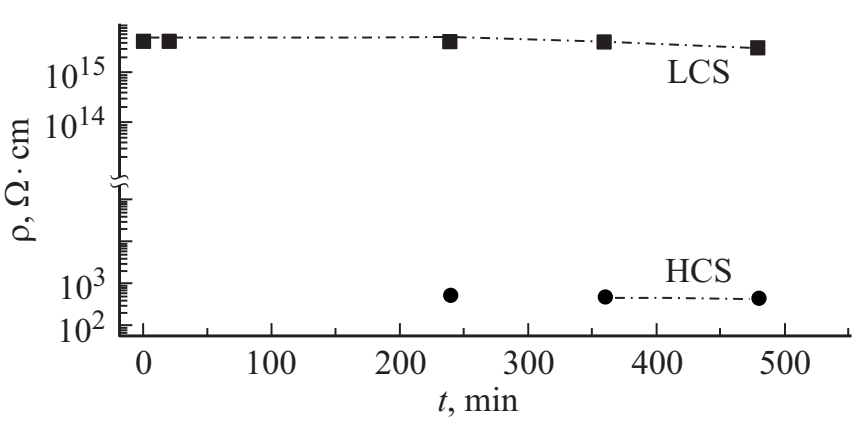

Рис. 2. Зависимость удельного сопротивления $\rho$ образцов от времени термолиза раствора ПВХ.

а в макромолекулах ПВХ образуются цепочки сопряженных двойных связей типа: ...- $\mathrm{C}=\mathrm{C}-\mathrm{C}=\mathrm{C}-\ldots$ случайной длины, которые имеют относительно слабо связанные $\pi$-электроны и фактически могут при определенных условиях превращаться в нанопроводники (размером $1-8 \mathrm{~nm}$ ). Используя описанный в $[11,12]$ процесс, нами был синтезирован полимер, содержащий сопряженные двойные связи (СДС) углерода на основе ПВХ, в макромолекулы которого встраиваются фрагменты молекул проводящего полимера-полиацетилена (ПАЦ) рис. 1. В зависимости от степени дегидрохлорирования, на начальной стадии процесса материал является классическим изолятором - широкозонным полимером. По мере увеличения концентрации цепочек сопряженных двойных связей углерода пленочные образцы, полученные из такого термолизованного ПВХ, моделируют композит с проводящими включениями и изолирующими промежутками.

Отметим также, что добавление фрагментов сопряженных двойных связей в процессе дегидрохлорирования ПВХ приводило к появлению в пленках определенных пластических свойств, так, что не было необходимости в использовании каких либо пластификаторов для изготовления достаточно эластичных для экспериментальных измерений пленочных образцов (от 10 до $200 \mu \mathrm{m})$. В наших экспериментах по исследованию электропроводности [12] такие сополимеры продемонстрировали самый высокий уровень проводимости в СВП по сравнению с другими исследованными ранее композитами ПВХ, в которых начальный уровень проводимости повышался введением специального пластификатора-модификатора А [8-10]. На рис. 2 приведена (в логарифмическом масштабе) зависимость удельного сопротивления пленочных образцов от времени термолиза. На рис. 2 хорошо видны два состояния электропроводности: состояние низкой проводимости CHП - LCS (Low conductivity State) и состояние высокой проводимости ВСП - HCS (High Conductivity State). График показывает, что удельное сопротивление образцов, начиная с некоторого уровня дегидрохлорирования, обнаруживает склонность к спонтанным переходам в СВП с изменением сопротивления на 13 и более 


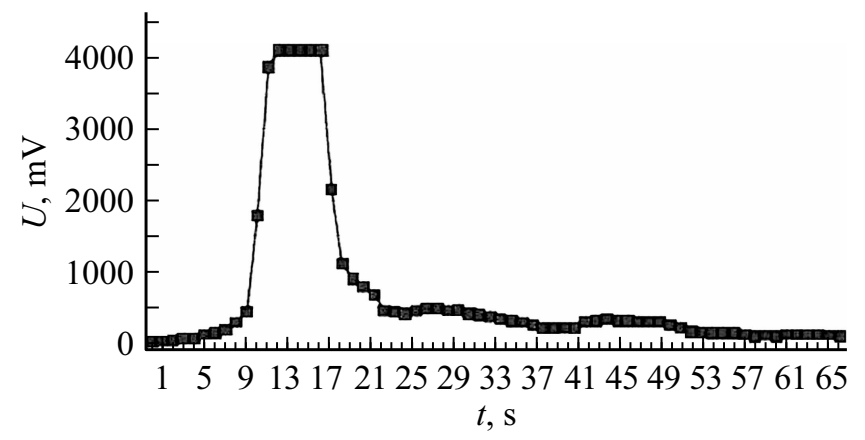

Рис. 3. Осциллограмма спонтанного перехода в СВП образца СДС полимера и его самопроизвольный возврат в СНП. Плоская вершина - ограничения АЦП.

порядков величины. Под термином „спонтанный“ здесь понимается переход в СВП „без видимых внешних воздействий“, т. е. при сохранении значений приложенного напряжения, температуры, давлении, освещении и т.д.

Высокий перепад удельных сопротивлений и хорошая воспроизводимость режимов спонтанных переходов, а также известная из литературы химическая структура материала, получаемого при дегидрохлорировании ПВХ, дают возможность на качественном уровне проанализировать физические механизмы наблюдаемых спонтанных скачков проводимости. Это позволяет строить качественные модели, описывающие наблюдаемые явления, в том числе наличие двух устойчивых состояний проводимости (точнее, относительную устойчивость состояния высокой проводимости (СВП)), а также широкий спектр наблюдаемых времен жизни СВП (от секунд до часов и суток). Полимерная специфика гигантских скачков проводимости в образцах с СДС углерода проявляется в том, что измеряемое сопротивление образцов через пленку падает до долей $\Omega$, но в то же время попытка измерить сопротивление вдоль пленки в ситуации, когда заведомо реализуется СВП, продемонстрировала отсутствие изменений поверхностного сопротивления пленки $R_{S}$ (более $10^{12} \Omega$ ), аналогично тому, что наблюдалось в пластифицированных ПВХ композитах [8-10]. При этом справедливое в случае однородного распределения токов выражение для сопротивления $R=\rho l / S$, где $\rho, l$ и $S$ удельное объемное сопротивление, длина и поперечное сечение образца соответственно, при разумном выборе параметров дает для $R_{S}$ оценку порядка $10 \mathrm{k} \Omega$, что очевидным образом не соответствует экспериментальным данным (в эксперименте $>10^{12} \Omega$ ) и свидетельствует в пользу сильной неоднородности распределения токов в образце. Фактически в отличие от нормальной проводимости наблюдаемые в образцах с СДС углерода скачки проводимости одномерны и направлены перпендикулярно пленке, т.е. возникающее СВП имеет сильную анизотропию (более $10^{9}$ ) на молекулярном уровне направленные микроканалы проводимости. Другой особенностью скачков проводимости в рассматриваемых ПВХ композитах можно считать широкий разброс про- должительности времен жизни СВП. Так на рис. 3 показана одна из реализаций случайного спонтанного перехода из состояния низкой проводимости (СНП) в СВП и после некоторого периода (около $10 \mathrm{~s}$ ) соответствующего обратного перехода в СНП. Отчетливо видно, что время жизни СВП существенно превышает времена переходов между состояниями.

\section{Анализ физического механизма аномальной проводимости}

В типичных схемах измерения проводимости изоляторов с большим балластным сопротивлением переход в СВП при увеличении напряжения реализуется в наших экспериментах такой обратимый переход, называемый нами мягкий „пробой“, наблюдался на всех ПВХ образцах при напряжениях порядка $30 \mathrm{~V} / \mu \mathrm{m}$, и на всех других полимерных образцах (полиэтилен, тефлон и т.д.). Этот эффект универсален, не зависит от типа полимера и описывается многочисленными теориями пробоя диэлектриков, т.е. высвобождением электронов из примесных атомов, мелких ловушек и дефектных узлов макромолекул, набором энергии во внешнем поле с дальнейшей ионизацией молекул основной матрицы. Термин „мягкий“ пробой мы применили к процессу
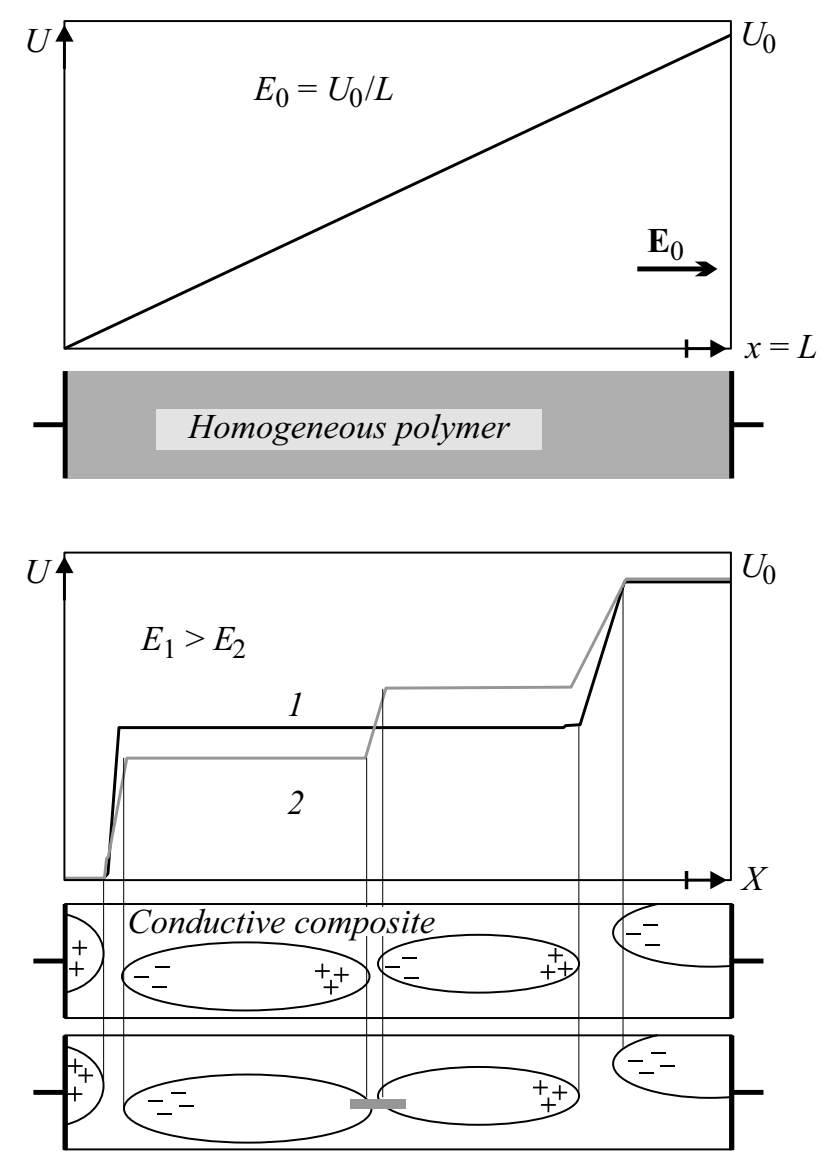

Рис. 4. Сравнение структуры поля в однородном (верхний график) и композитном материалах (рисунок снизу). 
пробоя, когда ток, возникающий при пробое, существенно ограничен балластным сопротивлением и энергия пробоя недостаточна для изменения структуры материала или образования необратимых изменений молекулярной структуры. Поэтому „мягкий“ пробой является неразрушающим и обратимым, в отличие от реального пробоя, где происходят необратимые изменения структуры образца за счет выделяемого тепла и искрового разряда. Все рассматриваемые в наших работах экспериментальные результаты по скачкам проводимости были получены при напряжениях, как минимум на порядок ниже порогов мягкого пробоя, причем в настоящей работе мы обсуждаем лишь спонтанные переходы, в то время как исследуемые обычно стимулированные переходы происходят под влиянием контролируемых внешних воздействий.

Для композитного материала с СДС, состоящего из проводящих элементов (нано- или микрочастиц), разделенных изолирующими промежутками, проводимость связана либо с туннелированием, либо с прыжками электронов между проводящими элементами композита. В отличие от „классической“ прыжковой проводимости, в которой носители заряда перескакивают с атома на атом, в случае композита такие прыжки имеют место между проводящими элементами, тогда как вдоль проводящего элемента заряды распространяются свободно. При этом возникает первое отличие, на которое следует обратить внимание, а именно существенное эффективное увеличение локальных значений электрического поля за счет введения проводящих элементов (рис. 4).

Если использовать упрощения выражения для вероятности $W$ туннелирования между двумя проводящими элементами, разделенными промежутком $\Delta x$, то можно записать:

$$
W\left(\Delta x, U_{0}\right)=W_{0} \exp \left(-\Delta x / l_{d}\right)
$$

где $1 / l_{d}=\left(8 m U_{0}\right)^{0.5} / \hbar, l_{d}-$ характеристическая длина, определяемая потенциальным барьером $U_{0}$ и эффективной массой заряда $m$. Если расстояние между электродами равно $L$, то можно ввести эффективную суммарную длину проводящих элементов на траектории прохождения заряда от одного электрода до другого $L_{C}$ , и тогда разность $L-L_{C}$ будет по порядку величины соответствовать эффективной длине изолирующих промежутков. Для получения приблизительной оценки вероятности туннелирования заряда с одного электрода на другой $W_{12}$ путем статистически независимых прыжков по одной из возможных траекторий между электродами запишем выражение для полной вероятности в качестве произведения вероятностей для каждого отдельного промежутка:

$$
W_{12}=\Pi^{W i}=W \exp \left(-\left(L-L_{C}\right) / l_{d}\right)
$$

Полный ток будет определяться суммой всех вероятностей (2) по всем траекториям, однако очевидно, что экспоненциальный множитель, в каждом слагаемом

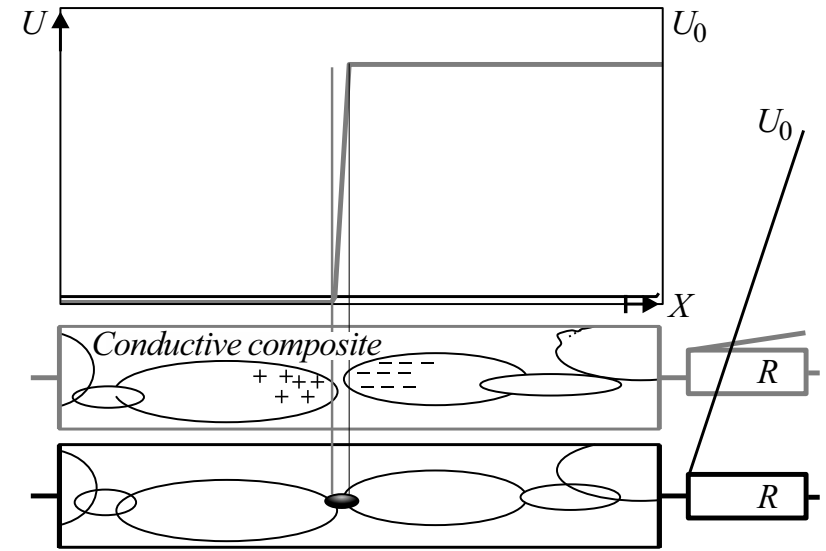

Pис. 5. Устойчивость проводящего состояния в композитном материале. В нижней части рисунка показана диаграмма распределения потенциала в СВП образца (все напряжение падает на балластном сопротивлении, см. справа); при флуктуационном переходе одного из проводящих элементов в СНП все напряжение падает на изолирующем мостике.

сократит сумму до нескольких членов с минимально короткими (суммарно) изолирующими промежутками. Таким образом, исходя из (2), можно считать, что ток будет протекать лишь в нескольких наиболее „коротких“ каналах и при определенных условиях может приводить к экспоненциальной зависимости проводимости от длины образца. С другой стороны, из выражения (2) следует, что при прохождении порога перколяции, т.е. после ,замыкании“ цепочки проводящих примесей, ток через образец композитного материала будет определяться проводимостью проводящих элементов. При этом должен наблюдаться скачек тока на несколько порядков величины. Очевидно, что при приближении композитного материала к порогу перколяции за счет увеличения концентрации проводящих примесей вероятность туннелирования и соответственно ток будут резко увеличиваться. Поэтому, именно в этой области можно ожидать необычного поведения проводимости. Действительно, можно показать, что именно вблизи порога перколяции композитная среда может быть неустойчивой относительно перехода в СВП, причем конкретные механизмы такого перехода, кроме туннелирования и прыжков носителей заряда, могут дополняться специфическими для данного композита механизмами переноса заряда. Для наглядности рассмотрим структуру поля в композитном образце, приведенную на рис. 4. Для однородного полимерного образца напряженность поля постоянна и его величина определяется как $E_{0}=U_{0} / L$. При добавлении проводящих элементов эффективное среднее электрическое поле сосредотачивается в изолирующих промежутках между проводниками:

$$
E=U /\left(L-L_{0}\right)
$$

и его значение будет увеличиваться по мере увеличения эффективной суммарной длины проводящих элементов. 


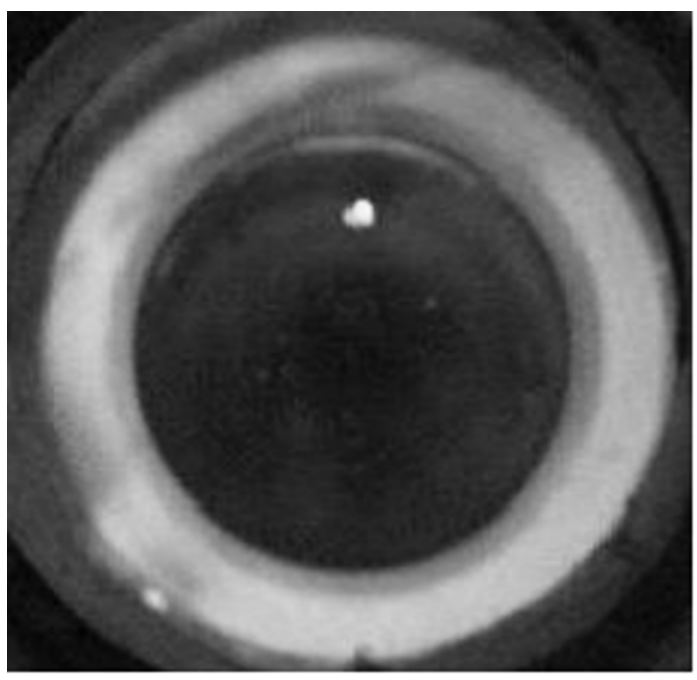

Рис. 6. Наблюдение вспышки светового излучения от токоведущего канала, возникающего в пленке образца с СДС при переходе в СВП. Размер кольца затемнения образца $40 \mathrm{~mm}$. Оценка толщины канала, требует специальных измерений, поскольку в данном случае использована камера с автофокусом.

В описываемых экспериментах с различными концентрациями проводящих элементов наблюдался заметный ток даже намного ниже порога перколяции. Поэтому, если в композите может быть реализован какой-либо физический механизм нелинейности, который может „замкнуть“ два соседних проводящих элемента (например, всегда есть вероятность мягкого пробоя), то поле в соседних изолирующих промежутках увеличится. И уже в следующем промежутке может произойти соответствующий переход в проводящее состояние и т.д. Следовательно, получаем, что композитная среда обладает неустойчивостью перехода в проводящее состояние, причем эта неустойчивость тем сильнее, чем ближе заполнение полимера проводящими элементами к порогу перколяции. Наличие неустойчивости каскадного замыкания изолирующих промежутков объясняет часть наблюдаемых результатов, т.е. относительно быстрый переход, в том числе спонтанный, в СВП за время существенно более короткое, чем времена жизни собственно СВП и СНП. По-прежнему остается вопрос: каков механизм устойчивости СВП. Для качественного ответа на этот вопрос обратимся к рис. 5, который в рамках предлагаемой гипотезы поясняет, каким образом в композитных материалах возникает устойчивость СВП.

В соответствии с развиваемой моделью, в СВП все перемычки между проводящими элементами замкнуты (например, вследствие мягкого пробоя). Если теперь (например, вследствие тепловых флуктуаций) один из элементов размыкается, то все электрическое поле скапливается именно в этом промежутке-изоляторе, который с большой вероятностью вновь испытывает мягкий пробой и становиться проводящим. Рассматриваемый каскадный механизм перескока в СВП универсален, т.е. не зависит от материала диэлектрика и механизма замыкания проводящих включений. Очевидно, что переход в СВП связан с вольт-амперной характеристикой и в случае спонтанных переходов такая характеристика должна быть функцией случайных параметров, а главное, должна иметь механизм памяти о предшествующем состоянии образца, что может приводить к гистерезису. Отметим, что при перескоках в СВП за счет внешне приложенного поля в отсутствие гистерезиса при снятии напряжения с образца СВП должно исчезать. Таким образом, наличие конечного и иногда достаточно продолжительного времени жизни и относительная устойчивость СВП за счет каскадного механизма слегка проясняют ситуацию с перескоками. Остается необъясненным тот экспериментальный факт, что образец ПВХ композита может сохранять состояние проводимости даже при снятии внешнего поля, в том числе на достаточно длительных временных интервалах порядка минут, часов и более [24]. Для объяснения такого поведения можно снова привлечь процесс дегидрохлорирования, т. е. происходящего при достаточно существенном нагревании отделения от макромолекулы ПВХ атомов водорода и хлора с образованием в макромолекуле двойной связи, что способствует резкому увеличению проводимости [25].

В отличие от термолиза за счет внешнего нагрева, о котором шла речь выше, в данном случае нагревание может происходить в результате выделения джоулева тепла непосредственно в толще образца при прохождении тока через проводящий канал. При этом будет эффективно увеличиваться концентрация сопряженных двойных связей углерода в области изолирующего промежутка и соответственно уменьшается его сопротивление т.е. возникает замыкание. Важно, что если этот процесс дегидрохлорирования происходит внутри твердого образца ПВХ, то движение „квазимолекулы“ $\mathrm{HCl}$, отделившейся от макромолекулы полимера, ограниченно свободным объемом с размерами порядка нанометров. Находясь в непосредственной близости от сопряженной двойной связи углерода, такая „квазимолекула“ может вновь войти в структуру макромолекулы ПВХ, превращая ее в изолятор. Такой механизм обратимого локального дегидрохлорирования может, в частности, порождать гистерезис, связанный с химической задержкой восстановления проводимости. Наличие проводящих каналов при переходе в СВП в образцах с СДС наблюдалось экспериментально при комнатной температуре $t=24^{\circ} \mathrm{C}$. Был использован специальный прозрачный электрод (слой ITO на стеклянной подложке) с проводимостью $100 \Omega$ на квадрат. При переходе в СВП образца с СДС (полученного дегидрохлорированием в течение $480 \mathrm{~min}$ ) через прозрачный электрод можно было при специальном затемнении наблюдать несколько слабосветящихся точек, которые на наш взгляд связаны с узкими токоведущими каналами и аналогичны наблюдениям, описанным и в других публикациях по исследованию электропроводности полимерных пленок [20-23]. Интен- 
сивность светящихся точек была различной и изменялась со временем, причем в некоторых каналах развивалось кратковременное до $10 \mathrm{~s}$ интенсивное свечение, величина которого была достаточной для уверенной регистрации фотоаппаратом. Характерная „вспышка“ канала показана на рис. 6.

Для получения грубой численной оценки локального разогрева полимерной пленки толщиной $10 \mu \mathrm{m}$ в условиях, близких к экспериментальным $[8,11,12]$, подсчитаем разогрев вещества в таком токоведущем канале. Будем считать, что полная мощность источника, нагрева токоведущий канал, диссипирует в объеме ПВХ за счет теплопроводности через стенки канала за время $t \sim S_{\perp} / \chi$, где $S_{\perp}$ - площадь поперечного сечения канала, а $\chi$ - коэффициент температуропроводности, связанный с теплопроводностью $\kappa$, плотностью $\rho_{\text {PVC }}$ и теплоемкостью $C_{\text {РVC }}$ известным соотношением $\chi=\kappa /\left(\rho_{\mathrm{PVC}} / C_{\mathrm{PVC}}\right)$. В результате для температуры нагрева канала получаем оценку

$$
\Delta T \sim(U \cdot I) /\left(\rho_{\mathrm{PVC}} C_{\mathrm{PVC}} V\right) \cdot\left(S_{\perp} / \chi\right)=(U \cdot I) /(\kappa L) .
$$

Подставляя сюда $U=15 \mathrm{~V}, \quad I=10^{-5} \mathrm{~A}, \quad \kappa=$ $=0.15 \mathrm{~W} /(\mathrm{mK}), \quad L=10^{-5} \mathrm{~m}, \quad$ получаем оценку изменения температуры канала $\Delta T \sim 100^{\circ} \mathrm{C}$. Таким образом, по порядку величины мы попадаем в диапазон температур начала активного дегидрохлорирования ПВХ (от 100 до $200^{\circ} \mathrm{C}$ ). Резкое уменьшение сопротивления токоведущего канала может привести к соответствующему увеличению мощности, возбуждению электролюминесценции, которые можно наблюдать экспериментально.

Разумеется, наиболее критическим для данной оценки является использование макроскопических, т.е. усредненных характеристик ПВХ, тогда как реально развитие неустойчивостей происходит на малых масштабах, где среда является сильно неоднородной, что должно приводить к сильным флуктуациям напряженности поля, проводимости, а также геометрических характеристик канала. Как следствие, выделение тепла в канале должно иметь неоднородный характер с максимумами в областях наименьшей проводимости. С другой стороны, и напряжения, прикладываемые к образцу, и ток также изменялись в экспериментах в достаточно широких пределах, так что порядок оценки изменения температуры в канале может попадать в диапазон локального развития реакции дегидрохлорирования.

\section{Заключение}

В работе была развита качественная модель наблюдаемых в ПВХ-композитах перескоков, позволяющая объяснить обнаруженные ранее в пленочных образцах (толщиной более $10 \mu \mathrm{m}$ ) частично дегидрохлорированного ПВХ обратимые переходы из состояния низкой проводимости в состояние высокой проводимости с амплитудой скачков до 13 порядков. С этой целью расширена предложенная ранее универсальная модель каскадного перехода композитного материала в СВП, и указаны механизмы неустойчивости, обеспечивающей относительно быстрые переходы образца из СНП в СВП и обратно. Для ПВХ композитов (и ему подобных полимеров, например, поливинилбромида) указан более низкопороговый по сравнению с мягким пробоем механизм перехода в СВП в рамках той же каскадной модели. Предложен структурно-химический механизм, объясняющий относительно длительные времена жизни СВП в ПВХ композитах, и основанный на том, что вблизи порога перколяции происходит заметный локальный разогрев ПВХ изолирующих промежутков, активизирующий (за счет изолированного элемента свободного объема) обратимую реакцию дегидрохлорирования с отделением „квазимолекулы“ $\mathrm{HCl}$, движение которой ограничено свободным объемом. С практической точки зрения с учетом развиваемой модели более тщательное исследование распределения времен жизни СВП может быть использовано для изучения свойств и структуры свободного объема в ПВХ композитах, наряду или вместо достаточно дорогого и сложного электрон-позитронного метода.

\section{Конфликт интересов}

Авторы заявляют, что у них нет конфликта интересов

\section{Список литературы}

[1] Barford W. // Electronic and Optical Properties of Conjugated Polymers, Clarendon, Oxford, 2005.

[2] Wan M. // Conducting Polymers with Micro or Nanometer Structure. Springer, 2008.

[3] Лачинов А.Н., Воробьева Н.В. // УФН. 2006. № 17. C. $1249-1266$

[4] Friedrich K., Fakirov S., Zhang Z. // Polymer Composites. From Nano-to Macro-Scale. Springer, 2005.

[5] Mai Eds. Y.-W., Yu Z.-Z. // Polymer nanocomposites. Woodhead Publ. Lim., Cambridge, 2006.

[6] Долбин И.В., Козлов Г.В., Зайков Г.Е. Структурная стабилизация полимеров: фрактальные модели. Изд-во Академия Естествознания, 2007.

[7] Pethrick R.A. Polymer Structure Characterization: From Nano to Macro Organization in Small Molecules and Polimers Ed. Royal Society of Chemistry, 2013.

[8] Власов Д.В., Крыштоб В.И., Власова Т.В., Апресян Л.А., Расмагин С.И. // Высокомолекулярные соединения. Серия А. 2015. Т. 57. № 3. С. 242. DOI: $10.7868 / \mathrm{S} 2308112015030165$

[9] Rasmagin S.I., Krasovskii V.I., Vlasov D.V., Apresyan L.A., Vlasova T.V., Kryshtob V.I., Feofanov I.N., Kazaryan M.A. // Proc. SPIE. 2015. Vol. 9810. DOI: 10.1117/12.2224963

[10] Крыштоб В.И., Расмагин С.И. // ЖТФ. 2017. Т. 87. Вып. 11. C. $1687-1689$.

DOI: $10.21883 / J T F .2019 .12 .48494 .220-18$

[11] Крыштоб В.И., Власов Д.В., Миронов В.Ф., Апресян Л.А., Власова Т.В., Расмагин С.И., Кураташвили З.А., Соловский А.A. // Электротехника. 2015. № 8. С. 39-42. 
[12] Крыштоб В.И., Власов Д.В., Миронов В.Ф., Апресян Л.А., Власова Т.В., Расмагин С.И., Кураташвили З.А., Соловский А.А. // Электротехника. 2014. № 5. С. 60-64.

[13] Передереева С.И., Орлов И.Г., Черкашин М.И. // Успехи химии. 1975. 44. С. 602-621.

[14] Шкловский Б.И., Эфрос А.Л. Электронные свойства легированных полупроводников. М.: Наука, 1979.

[15] Stallinga P. // Adv. Mater. 2011. Vol. 23. P. 3356-3362.

[16] Sheng P. // Phys. Rev. 1980. Vol. 21. P. 2180-2195.

[17] Ландау Л.Д., Лифшии, Е.М. Квантовая механика. Теоретическая физика. Т. III. М.: Физматлит, 2004.

[18] Чопра К.Л. Электрические явления в тонких пленках. М.: Мир, 1972.

[19] Ламперт М., Марк П. Инжекционные токи в твердых телах. М.: Мир, 1973.

[20] Ovshinsky S.R. // Phys. Rev. Lett. 1968. Vol. 21. P. $1450-1453$.

[21] Скалдин О.А., Жеребов А.Ю., Лачинов А.Н., Чувыров А.Н., Делев В.А. // Письма в ЖЭТФ. 1990. Т. 51. С. 141-144.

[22] Ениколопян Н.С., Берлин Ю.А., Бешенко С.И., Жорин В.А. // Письма в ЖЭТФ. 1981. Т. 33. С. 508-511.

[23] Берлин Ю.А., Бешенко С.И., Жорин В.А. // ДАН. 1981. T. 260. С. 1389-1390.

[24] Крыштоб В.И., Расмагин С.И., Власова Т.В. // Электротехника. 2018. № 6. С. 48-50.

[25] Rasmagin S.I., Krasovskii V.I., Novikov I. K., Novikov I.K., Fofanov I.N. Optical methods for controlling the degree dehydrochlorination of polyvinylchloride // Conference: XIII International Conference on Atomic and Molecular Pulsed Lasers Volume: 10614 P.4.2018. doi:10.1117/12.2302981 